\title{
Herida por proyectil de arma de fuego en cuello con lesión traqueoesofágica y colocación de parche muscular de esternotiroideo
}

\author{
Jorge Tadeo Palacios-Zertuche, Carlos Alberto Montero-Cantú, Armando de Jesús Guerrero-Hernández, \\ Rogelio Salinas-Domínguez, Edelmiro Pérez-Rodríguez, Gerardo Enrique Muñoz-Maldonado $₫$
}

Hospital Universitario “Dr. José Eleuterio González”, Universidad Autónoma de Nuevo León. Monterrey, Nuevo León, México. Trabajo recibido: 22-VI-2016; aceptado: 05-VIII-2016

\begin{abstract}
RESUMEN. Antecedentes: Las lesiones penetrantes del cuello representan del $5-10 \%$ de todos los casos de trauma que se presentan en el Servicio de Urgencias y dan como resultado una mortalidad significativa. Caso clínico: Masculino de 34 años de edad que acudió al Departamento de Urgencias por presentar herida por proyectil de arma de fuego en cuello. Se le realizó intubación endotraqueal y trasladado a quirófano. Por medio de una incisión cervical transversa se observaron lesión esofágica y traqueal a nivel del tercero y cuarto anillo, se colocó cánula de traqueostomía en orificio anterior de lesión traqueal, desbridando bordes de lesiones esofágicas y cerrando de forma primaria. Se tomó un colgajo vascularizado de esternotiroideo para colocar parche muscular entre la lesión traqueal y esofágica, dejando drenaje y realizando gastrostomía. Al paciente se le dio alimentación enteral por la gastrostomía, al décimo día se inició vía oral sin datos de fuga. Conclusión: Es imprescindible colocar un colgajo de músculo vascularizado de esternocleidomastoideo o un músculo infrahioideo para proteger la reparación esofágica y reducir la incidencia de fuga y fístula traqueoesofágica.
\end{abstract}

Palabras clave: Lesiones en cuello, lesiones traqueales, lesiones esofágicas, parche muscular.

ABSTRACT. Background: Penetrating neck injuries represent $5-10 \%$ of all trauma lesions that present to the emergency room resulting in significant mortality. Case report: 34 -year-old male is brought to the emergency room with a gunshot wound in the neck. Urgent endotracheal intubation was performed and then referred to the operating room. We performed a transverse cervical incision observing both, esophageal and tracheal lesions comprising the third and fourth tracheal rings. A tracheostomy tube was put in place through the anterior tracheal lesion. Debridement of the borders and primary closure of the esophageal lesion was carried out in order to place a vascularized sternothyroid muscle flap between the tracheal and esophageal injuries. A drain is placed and gastrostomy is performed. Enteral nutrition via gastrostomy was indicated for ten days. Oral intake was reinstituted afterwards without evidence of leakage. Conclusion: A vascularized muscle flap, involving the sternocleidomastoid or infrahyoid muscles, is critical to protect the esophageal repair and reduce the incidence of tracheoesophageal fistulas and leakage.

Key words: Neck injuries, tracheal injuries, esophagus injuries, muscle patch.

\section{ANTECEDENTES}

Las lesiones penetrantes del cuello representan alrededor del $5-10 \%$ de todos los casos de trauma que se presentan en el Servicio de Urgencias y dan como resultado una mortalidad significativa. ${ }^{1-3}$ Los componentes cervicales con más frecuencia lesionados en traumatismos penetrantes están relacionados con las lesiones vasculares (21-27\%), seguido de la médula espinal (16\%) y el tracto aero-digestivo (6-10\%), como la laringe o la tráquea y de la faringe o el esófago. ${ }^{1}$ Lo más común es que estas lesiones sean secundarias a heridas por arma blanca y de fuego. ${ }^{4}$

\section{CASO CLÍNICO}

Masculino de 34 años de edad que acudió al Departamento de Urgencias por presentar herida por proyectil de arma de fuego en cuello (figura 1). A su ingreso, en la exploración física se encontró herida de la entrada del proyectil en línea media del cuello en zona 1 y salida en zona 2 posterior izquierda, frecuencia cardíaca de 120 latidos por minuto, frecuencia respiratoria de 32 respiraciones por minuto, presión arterial de 130/80 $\mathrm{mmHg}$, saturación de oxígeno al $82 \%$ y sin compromiso neurológico. Se le realizó intubación endotraqueal y trasladó a quirófano. 
Por medio de una incisión cervical transversa fueron observadas lesión traqueal en espejo a nivel del tercer y cuarto anillo (figura 2) y lesión esofágica en espejo (figura 3); lo siguiente fue colocar cánula de traqueostomía en orificio anterior de lesión traqueal, desbridando bordes de lesiones esofágicas y cerrando de forma primaria. No presentó lesión vascular en columna cervical, ni en otras estructuras. Se procede a tomar un colgajo vascularizado de esternotiroideo colocando parche muscular entre la lesión traqueal y esofágica (figura 4), dejando drenaje y realizando gastrostomía. El paciente recibió alimentación vía enteral por la gastrostomía y al décimo día inició por vía oral sin datos de fuga retirando drenaje, dándose de alta al doceavo día posoperatorio. En el seguimiento por consulta y a las cuatro semanas del procedimiento quirúrgico retiramos sonda de gastrostomía y se decanula previa traqueoscopia, realizando una de control a los dos meses sin evidenciar estenosis. A los ocho meses de la cirugía no hay datos de estenosis traqueal ni de secuela en el esófago.

\section{DISCUSIÓN}

Las primeras descripciones del tratamiento de heridas penetrantes del cuello provienen de 1522, cuando Ambroise Paré describió la técnica de ligadura de los grandes vasos en el sitio de la lesión. Estos principios se utilizaron más tarde durante la Primera Guerra Mundial, la mortalidad por heridas en el cuello era de $60 \%$

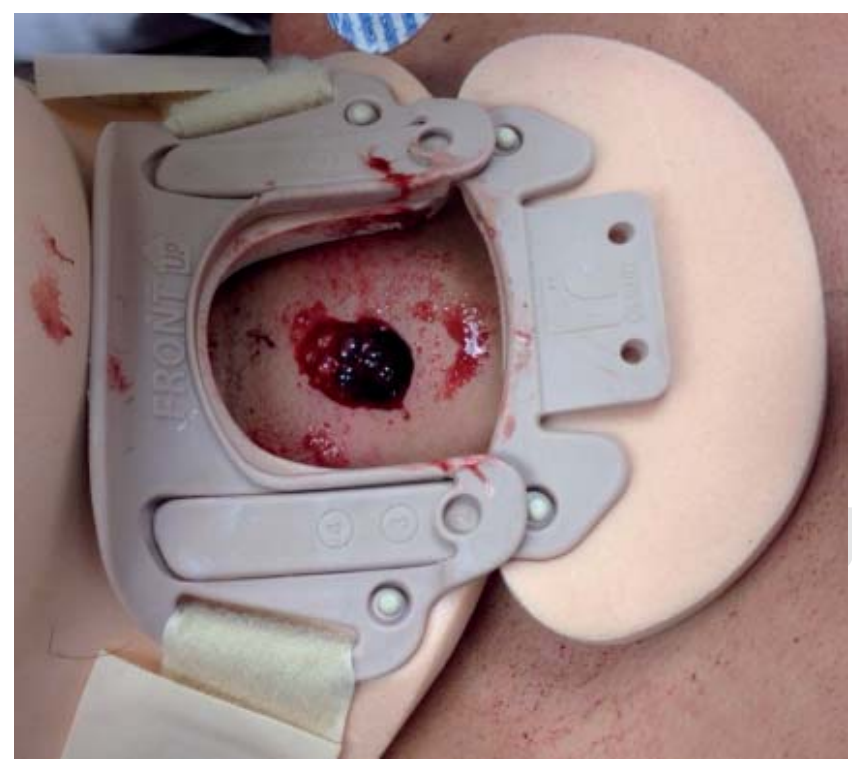

Figura 1. Orificio de herida por proyectil de arma de fuego en cuello. en ese tiempo. Durante la Segunda Guerra Mundial se estableció la revisión obligatoria de todas las heridas penetrantes en el cuello. ${ }^{5}$

Por definición, una herida penetrante en el cuello es aquella que atraviesa el espesor total del platisma, si el platisma está intacto la herida se considera superfi-

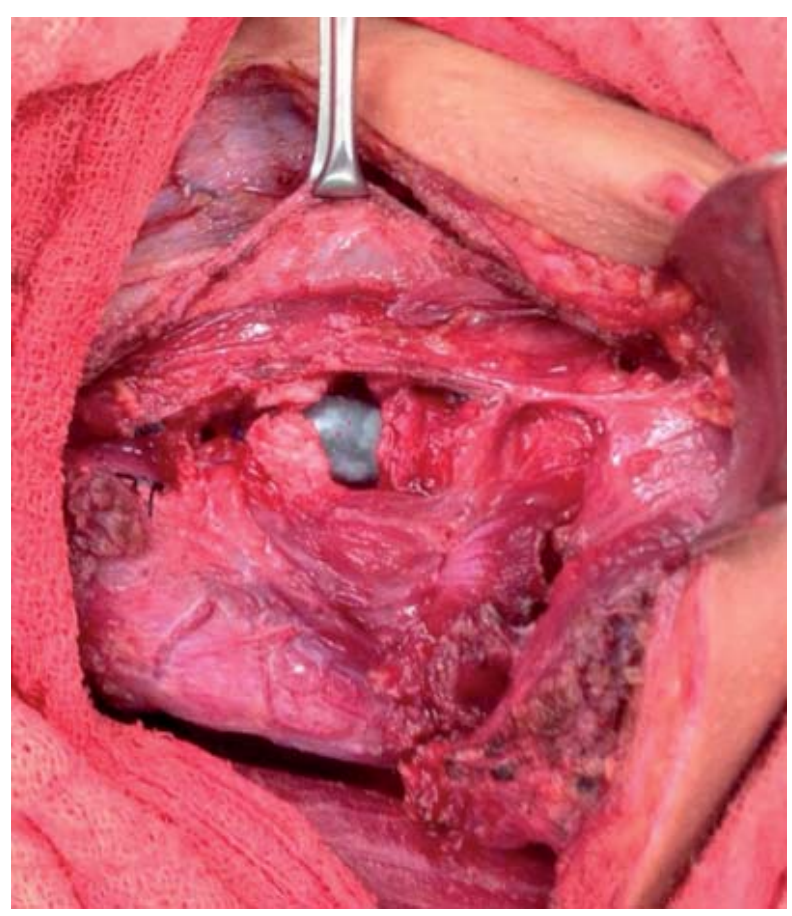

Figura 2. Lesión traqueal a nivel del tercer y cuarto anillo.

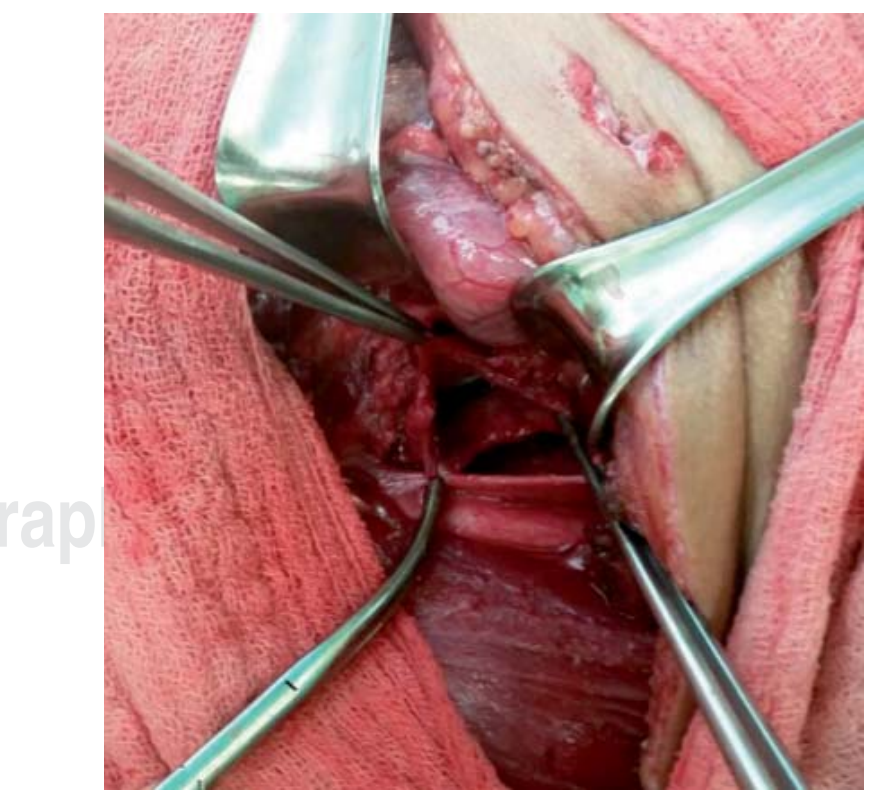

Figura 3. Lesión esofágica. 


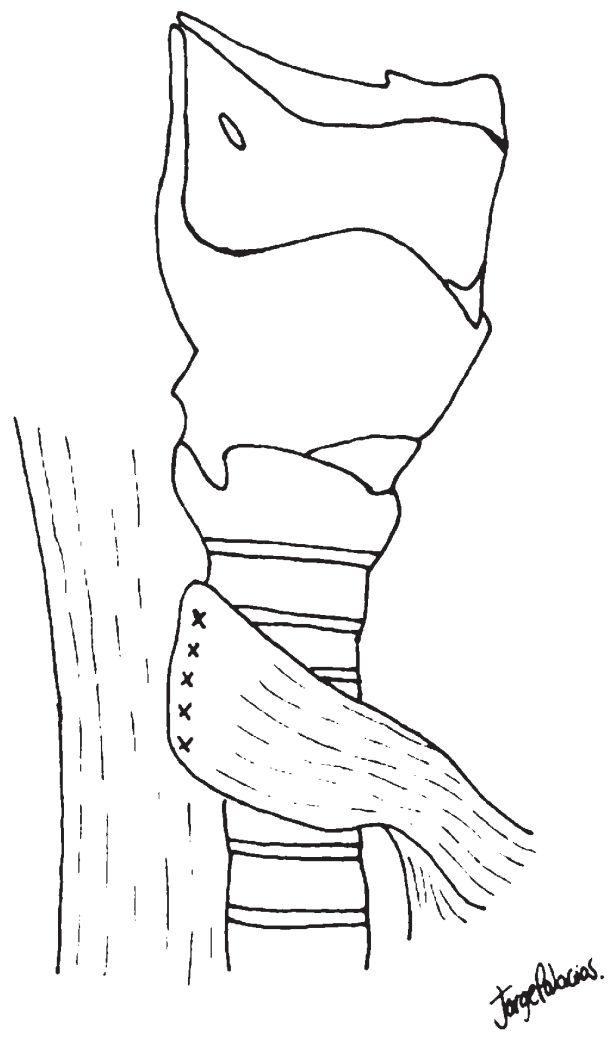

Figura 4. Parche muscular de esternotiroideo entre la lesión traqueal y esofágica.

cial. Las lesiones penetrantes del cuello se clasifican de acuerdo con el sitio de penetración en tres zonas anatómicas del cuello. La Zona I se extiende desde las clavículas hasta el borde inferior del cartílago cricoides. La Zona II se extiende desde el borde inferior del cartílago cricoides al ángulo de la mandíbula. La Zona III se extiende desde el ángulo de la mandíbula a la base del cráneo. ${ }^{4}$

La Zona I representa una zona peligrosa porque las estructuras vasculares están en estrecha proximidad al tórax. Una lesión en la Zona I tiene un índice alto de mortalidad (12\%). La exploración obligatoria no se recomienda para las lesiones de la Zona I, con frecuencia se sugiere la angiografía para asegurarse de que los grandes vasos no están lesionados. La Zona II es la región más comúnmente afectada ( 60 a $75 \%$ ), las lesiones en esta zona han creado una gran controversia en la literatura acerca del manejo en los últimos 15 años. Existe un debate en curso sobre el uso de la exploración obligatoria frente a la exploración selectiva con un examen serial, pruebas endoscópicas y angiografía. La Zona III está protegida por las estructuras esqueléticas y es difícil de estudiar debido a la base del cráneo y la necesidad de dividir o desplazar la mandíbula. ${ }^{6}$
En la actualidad la angio-TAC es un método no invasivo, eficaz y fácil disponibilidad para la evaluación de trauma en el cuello, lo que reduce de manera significativa el número de procedimientos quirúrgicos, así como la cervicotomía no terapéutica. Los estudios muestran que el angio-TAC tiene una alta capacidad diagnóstica ante todo, en la identificación de lesiones en las arterias y las venas del cuello, con una especificidad casi del $100 \%$ y sensibilidad del $90 \%$. Las lesiones traqueales son poco comunes dentro de las lesiones penetrantes del cuello y ocurren en $1-7 \%$ de los pacientes. ${ }^{7}$ El primer paso en el trauma penetrante del cuello es garantizar la seguridad de la vía aérea; y tres cuartas partes de los pacientes con lesión laringotraqueal necesitan una intervención para asegurarla mediante una intubación orotraqueal o una cricotiroidotomía emergente. ${ }^{8}$

Cuando hay pérdida de tejido en la parte anterior - la tráquea lateral, un tubo de traqueostomía puede colocarse en el defecto hasta tomar una decisión sobre el uso de un parche de músculo vascularizado o una reconstrucción formal. ${ }^{9}$

Los pacientes con lesiones traqueales y traqueostomisados pueden llegar a presentar estenosis traqueal de tipo adquirido presentando signos y síntomas propios de una obstrucción de la vía aérea alta, como disnea con el esfuerzo, sibilancias, estridor e incluso neumonías, por lo que el seguimiento en estos pacientes es primordial. ${ }^{10}$ En la valoración de los pacientes puede emplearse la laringotraqueoscopia, radiografías, TAC y la RMN. ${ }^{11}$ Sin embargo, la broncoscopia es el método diagnóstico de elección en los pacientes con patología estenosante de la vía aérea y es el procedimiento más fiable en la determinación del tipo de lesión, sus características, localización y extensión. ${ }^{10}$

La lesión traumática en el esófago es rara, representa del $0.9-6.6 \%$ de las lesiones penetrantes del cuello. Se ha demostrado que un retraso en el diagnóstico y en la intervención quirúrgica aumenta el número de complicaciones resultantes de la mediastinitis, absceso y sepsis. La detección temprana de lesiones esofágicas es imperativa y la mortalidad se estima de hasta un $20 \%{ }^{4}$

Las perforaciones del esófago cervical tienen un mejor pronóstico que las que incluyen otros niveles del esófago. El dolor de cuello, odinofagia, disfasia, enfisema subcutáneo, fiebre, eritema, debilidad, disfonía, ronquera y crepitación en el cuello son hallazgos casi siempre comunes después de una perforación del esófago cervical. ${ }^{2}$

Además, el uso de broncoscopia, esofagoscopia y estudios contrastados con fluoroscopia también pueden ser extremadamente útiles. La examinación endoscópica y radiológica combinada del esófago en particular 
se sabe que mejora la sensibilidad que cada prueba de forma aislada. ${ }^{12}$

La lesión traumática del esófago por lo común se diagnostica por tomografía computarizada o en el quirófano. La tasa de mortalidad es casi de $29 \%$ y la morbilidad es al menos del $47 \%{ }^{8}$ El éxito de la reparación primaria dentro de las primeras 24 horas de la perforación esofágica se evidencia en varios estudios. ${ }^{2,9}$ Un esofagograma contrastado con Gastrografin o bario tiene una sensibilidad menor al $100 \%$ en el diagnóstico, y la esofagoscopia flexible se realiza en pacientes con alto riesgo con estudios contrastados negativos. La combinación de un estudio de contraste y la esofagoscopia tiene una exactitud de casi $100 \%$ en los pacientes con lesiones esofágicas en la Zona II. ${ }^{9}$ Una esofagostomía cervical con gastrostomía o yeyunostomía han sido empleados en pacientes con extensa contaminación mediastinal, necrosis esofágica severa u obstrucción patológica, esófago ampliamente desvitalizado o inestabilidad hemodinámica incapaces de tolerar la reparación o resección definitiva. ${ }^{2}$

Se han utilizado colgajos de músculo, pleura y pericardio para ayudar a la curación y proteger las líneas de sutura en lesiones de la tráquea y esófago así como en reparaciones traqueoesofágicas concomitantes. ${ }^{13}$

Una tasa de complicaciones posoperatorias del $74 \%$ se informó en antiguas series de 23 lesiones traqueoesofágicas combinadas. El análisis de las complicaciones documenta que la mayoría eran debido a fugas de las reparaciones del esófago. Cuando existen reparaciones adyacentes de la tráquea y el esófago, la tráquea y la arteria carótida, o la arteria carótida y el esófago, un colgajo vascularizado del músculo esternocleidomastoideo debe ser envuelto alrededor de la reparación visceral. Esto reduce la incidencia de una fuga de la reparación visceral y si se produce una fuga, proteger a la reparación arterial adyacente..$^{9}$ En lesiones traqueoesofágicas concomitantes este colgajo es realizado seccionando la cabeza esternal del músculo esternocleidomastoideo a nivel de su inserción, se rota medialmente, se coloca entre la reparación traqueoesofágica y se fija con puntos separados. ${ }^{14}$

En el esófago cervical pueden emplearse parches musculares (esternohioideo, esternotiroideo o esternocleidomastoideo) para reforzar reparaciones, y entre el esófago y la tráquea en caso de lesión combinada. En nuestro paciente optamos por realizar un colgajo muscular de esternotiroideo, músculo irrigado por la arteria tiroidea superior e inferior en su región cefálica y caudal, respectivamente, seccionando transversal y rotando la porción inferior medialmente, colocándolo entre la tráquea y el esófago y fijándolo con puntos separados de sutura absorbible.

\section{CONCLUSIONES}

En las lesiones penetrantes del cuello que comprometen la tráquea y el esófago, lo primordial es establecer una vía aérea ya sea con intubación orotraqueal o quirúrgica dependiendo de cada caso en particular. Si se decide realizar cierre primario de la lesión esofágica y colocación de una cánula de traqueostomía o reparación de la lesión traqueal, es imprescindible colocar un colgajo de músculo vascularizado de esternocleidomastoideo o un músculo infrahioideo para proteger la reparación esofágica y reducir la incidencia de fuga y fístula traqueoesofágica.

\section{REFERENCIAS}

1. Nasr A, de Oliveira JT, Mazepa MM, et al. Evaluation of the use of tomography in penetrating neck trauma. Rev Col Bras Cir 2015;42(4):215-219. doi: 10.1590/010069912015004004.

2. Varghese A. Penetrating neck injury: a case report and review of management. Indian J Surg 2013;75(1):43-46. doi: 10.1007/s12262-012-0531-7.

3. Ball CG. Penetrating nontorso trauma: the head and the neck. Can J Surg 2015;58(4):284-285.

4. Saito N, Hito R, Burke PA, Sakai O. Imaging of penetrating injuries of the head and neck: current practice at a level I trauma center in the United States. Keio J Med 2014;63(2):23-33.

5. Makhani M, Midani D, Goldberg A, Friedenberg FK. Pathogenesis and outcomes of traumatic injuries of the esophagus. Dis Esophagus 2014;27(7):630-636. doi: 10.1111/dote.12132.

6. Łochowski M, Kaczmarski J, Brzeziński D, CieCieślikWolski B, Kozak J. Penetrating neck traumas. Kardiochir Torakochirurgia Pol 2014;11:30-33. doi: 10.5114/ kitp.2014.41927.

7. Aghajanzadeh M, Porkar NF, Ebrahimi H. Cervical esophageal perforation: a 10-year clinical experience in north of iran. Indian J Otolaryngol Head Neck Surg 2015;67(Suppl 1):34-39. doi: 10.1007/s12070-014-0737-z.

8. Ozbilen Acar G, Tekin M, Cam OH, Kaytanci E. Larynx, hypopharynx and mandible injury due to external penetrating neck injury. Ulus Travma Acil Cerrahi Derg 2013;19(3):271-273. doi: 10.5505/ tjtes.2013.58259.

9. Mattox KL. Neck. En: Feliciano DV, Vercruysse GA, editores. Trauma. USA: McGraw Hill; 2015. p. 421, 426.

10. Barbancho DC, Antón-Pacheco J, Díaz ML, Sánchez RT, García JC, Fraile AG. Acquired tracheal stenosis: diagnosis and treatment. Cir Pediatr 2007;20(1):19-24.

11. Nair S, Mohan S, Mandal G, Nilakantan A. Tracheal stenosis: our experience at a tertiary care centre in India 
with special regard to cause and management. Indian J Otolaryngol Head Neck Surg 2014;66(1):51-56. doi: 10.1007/s12070-013-0663-5.

12. Hope N, Gray G, Lesay M, Reddy E. Penetrating neck injuries: the point of plain films. Clin Case Rep 2016;4:216218. doi: 10.1002/ccr3.481.

13. Victorino GP, Porter JM, Henderson VJ. Use of a gastric pull-up for delayed esophageal reconstruction in a patient with combined traumatic injuries of the trachea and esophagus. J Trauma 2000;49(3):563-564.

14. Losken A, Rozycki GS, Feliciano DV. The use of the sternocleidomastoid muscle flap in combined injuries to the esophagus and carotid artery or trachea. J Trauma 2000;49(5):815-817.

\section{$\triangle$ Correspondencia:}

Dr. Gerardo Enrique Muñoz-Maldonado

Avenida Francisco I. Madero y Gonzalitos s/n,

Colonia Mitras Centro, 64460,

Monterrey, Nuevo León, México.

Teléfono: (81) 83483303

Correo electrónico: cevam99@gmail.com jorgepalacios_@hotmail.com

Los autores declaran no tener conflicto de intereses. 